\title{
On differential independence of the Riemann zeta function and the Euler gamma function
}

by

\section{Bao Qin Li (Miami, FL) and Zhuan Ye (DeKalb, IL)}

It is well-known ([2] and [4]) that $\Gamma$, as well as $\zeta$, is not a solution of any algebraic differential equation with coefficients in $\mathbb{C}$. In other words, if $P\left(u_{0}, u_{1}, \ldots, u_{m}\right)$ is any polynomial in $u_{0}, u_{1}, \ldots, u_{m}$ over $\mathbb{C}$, and

$$
P\left(\Gamma, \Gamma^{\prime}, \ldots, \Gamma^{(m)}\right)(z) \equiv 0 \quad \text { or } \quad P\left(\zeta, \zeta^{\prime}, \ldots, \zeta^{(m)}\right)(z) \equiv 0,
$$

for all $z \in \mathbb{C}$, then the polynomial $P$ is identically zero. This answers Hilbert's conjecture [1] in his 18th problem. For a detailed discussion of this subject and other related topics, we refer the reader to [5].

Let $h(z)=\zeta(\sin (2 \pi z))$. Recently, Markus [3] proved that if

$$
P\left(h, h^{\prime}, \ldots, h^{(m)} ; \Gamma, \Gamma^{\prime}, \ldots, \Gamma^{(n)}\right)(z) \equiv 0 \quad \text { for } z \in \mathbb{C},
$$

then the polynomial $P\left(u_{0}, u_{1}, \ldots, u_{m} ; v_{0}, v_{1}, \ldots, v_{n}\right)$ is identically zero. Thus, in the terminology of differential algebraic theory, $\Gamma$ and $h$ are differentially independent over $\mathbb{C}$ (hence, over $\mathbb{C}(z)$ ). Furthermore, Markus [3] conjectured that if

$$
P\left(\zeta, \zeta^{\prime}, \ldots, \zeta^{(m)} ; \Gamma, \Gamma^{\prime}, \ldots, \Gamma^{(n)}\right)(z) \equiv 0 \quad \text { for } z \in \mathbb{C},
$$

then the polynomial $P\left(u_{0}, u_{1}, \ldots, u_{m} ; v_{0}, v_{1}, \ldots, v_{n}\right)$ is identically zero, i.e. $\Gamma$ and $\zeta$ are differentially independent. In this short note, we prove that $\zeta$ and $\Gamma$ cannot satisfy a class of algebraic differential equations.

Let $P\left(u_{0}, u_{1}, \ldots, u_{m} ; v_{0}, v_{1}, \ldots, v_{n}\right)$ be any polynomial with coefficients in $\mathbb{C}$. For a non-negative integer $\mu$, we let

$$
\begin{array}{r}
\Lambda=\Lambda(\mu)=\left\{\left(\lambda_{0}, \lambda_{1}, \ldots, \lambda_{\mu}\right): \lambda_{j}\right. \text { is a non-negative integer } \\
\text { and } 0 \leq j \leq \mu<\infty\}
\end{array}
$$

2000 Mathematics Subject Classification: Primary 33B15; Secondary 12H05, 30D35, $34 \mathrm{M} 15$.

Key words and phrases: the Riemann zeta-function, the Euler gamma-function, algebraic differential equation. 
be a finite index set. Define

$$
\begin{aligned}
&|\lambda|=\sum_{j=0}^{\mu} \lambda_{j} \quad \text { and } \quad \Lambda_{k}=\{\lambda \in \Lambda:|\lambda|=k\} ; \\
&|\lambda|_{*}=\sum_{j=0}^{\mu} j \lambda_{j} \quad \text { and } \quad \Lambda_{k}^{*}=\left\{\lambda \in \Lambda:|\lambda|_{*}=k\right\} .
\end{aligned}
$$

Thus, there is a non-negative integer $N$ such that

$$
P\left(u_{0}, u_{1}, \ldots, u_{m} ; v_{0}, v_{1}, \ldots, v_{n}\right)=\sum_{j=0}^{N} \sum_{\lambda \in \Lambda_{j}} a_{\lambda}\left(u_{0}, \ldots, u_{m}\right) v_{0}^{\lambda_{0}} v_{1}^{\lambda_{1}} \cdots v_{n}^{\lambda_{n}}
$$

where $a_{\lambda}\left(u_{0}, u_{1}, \ldots, u_{m}\right)$ is a polynomial in $u_{0}, u_{1}, \ldots, u_{m}$ with coefficients in $\mathbb{C}$. Set, for $j=0,1, \ldots, N$,

$$
P_{j}\left(u_{0}, u_{1}, \ldots, u_{m} ; v_{0}, v_{1}, \ldots, v_{n}\right)=\sum_{\lambda \in \Lambda_{j}} a_{\lambda}\left(u_{0}, \ldots, u_{m}\right) v_{0}^{\lambda_{0}} v_{1}^{\lambda_{1}} \cdots v_{n}^{\lambda_{n}} .
$$

For simplicity, we write $P\left(u_{0}, u_{1}, \ldots, u_{m} ; v_{0}, v_{1}, \ldots, v_{n}\right)$ as $P(u ; v)$ if it does not cause confusion, and similarly for $P_{j}(u ; v)$. Further, we write

$$
P_{j}(u ; v)=\sum_{p=0}^{M_{j}} \sum_{\lambda \in \Lambda_{j} \cap \Lambda_{p}^{*}} a_{\lambda}\left(u_{0}, \ldots, u_{m}\right) v_{0}^{\lambda_{0}} v_{1}^{\lambda_{1}} \cdots v_{n}^{\lambda_{n}},
$$

where $M_{j}$ is a non-negative integer and, in the same manner,

$$
P_{j, p}(u ; v)=\sum_{\lambda \in \Lambda_{j} \cap \Lambda_{p}^{*}} a_{\lambda}\left(u_{0}, \ldots, u_{m}\right) v_{0}^{\lambda_{0}} v_{1}^{\lambda_{1}} \cdots v_{n}^{\lambda_{n}} .
$$

Consequently, we obtain

$$
\begin{aligned}
P_{j}(u ; v) & =\sum_{p=0}^{M_{j}} P_{j, p}(u ; v) \\
P(u ; v) & =\sum_{j=0}^{N} P_{j}(u ; v)=\sum_{j=0}^{N} \sum_{p=0}^{M_{j}} P_{j, p}(u ; v) .
\end{aligned}
$$

Theorem. Let $P(u ; v)$ be a non-trivial polynomial defined as in (3). If

$$
\sum_{\lambda \in \Lambda_{j} \cap \Lambda_{p}^{*}} a_{\lambda}\left(u_{0}, \ldots, u_{m}\right) \not \equiv 0 \quad \text { whenever } \quad P_{j, p}(u ; v) \not \equiv 0,
$$

for all possible $j$ 's and $p$ 's, then

$$
P\left(\zeta, \zeta^{\prime}, \ldots, \zeta^{(m)} ; \Gamma, \Gamma^{\prime}, \ldots, \Gamma^{(n)}\right)(z) \not \equiv 0 .
$$

To prove our theorem, we need the following celebrated theorem. 
Lemma (Voronin, [7]). For any $1 / 2<\sigma<1$, the curve $(\zeta(\sigma+i t)$, $\left.\zeta^{\prime}(\sigma+i t), \ldots, \zeta^{(m)}(\sigma+i t)\right),-\infty<t<\infty$, is everywhere dense in $\mathbb{C}^{m+1}$.

Proof of Theorem. If $N=0$, then $P(u ; v)$ is a polynomial in $u_{0}, u_{1}, \ldots, u_{m}$ only; denote it by $P_{0}(u)$. If $P_{0}(u)=P_{0,0}(u) \neq 0$ at a point $w_{*}=\left(w_{0}, w_{1}\right.$, $\left.\ldots, w_{m}\right) \in \mathbb{C}^{m+1}$, then there is a neighborhood $U$ of $w_{*}$ such that $P_{0}(u) \neq 0$ for $u \in U \subset \mathbb{C}^{m+1}$. By Voronin's lemma, there is a sequence of positive real numbers $t_{q}$ such that

$$
\left(\zeta, \zeta^{\prime}, \ldots, \zeta^{(m)}\right)\left(3 / 4+i t_{q}\right) \in U
$$

for all large $q$. Therefore,

$$
P_{0}\left(\zeta, \zeta^{\prime}, \ldots, \zeta^{(m)}\right)\left(3 / 4+i t_{q}\right) \neq 0
$$

for all large $q$ and the theorem is proved in this case.

We now assume that $N$ in (3) is greater than zero. Since $P(u ; v)$ is nontrivial, we can assume that $P_{j_{0}}(u ; v)$ is the first one that is not identically zero in the sequence

$$
P_{0}(u ; v), P_{1}(u ; v), \ldots, P_{N}(u ; v),
$$

and that $P_{j_{0}, p_{0}}(u ; v)$ is the first one that is not identically zero in the sequence

$$
P_{j_{0}, M_{j_{0}}}(u ; v), P_{j_{0}, M_{j_{0}}-1}(u ; v), \ldots, P_{j_{0}, 0}(u ; v) .
$$

Without loss of generality, we may assume that $c=j_{0}$ and $d=M_{j_{0}}$ for simplicity. It is clear from (3) that

$$
P\left(\zeta, \zeta^{\prime}, \ldots, \zeta^{(m)} ; \Gamma, \Gamma^{\prime}, \ldots, \Gamma^{(n)}\right)(z) \not \equiv 0
$$

if and only if

$$
\begin{aligned}
& \frac{P\left(\zeta, \zeta^{\prime}, \ldots, \zeta^{(m)} ; \Gamma, \Gamma^{\prime}, \ldots, \Gamma^{(n)}\right)(z)}{\Gamma^{N}(z)} \\
& \quad=\sum_{j=0}^{N} \frac{1}{\Gamma^{N-j}(z)} \sum_{p=0}^{M_{j}} P_{j, p}\left(\zeta, \zeta^{\prime}, \ldots, \zeta^{(m)} ; 1, \Gamma^{\prime} / \Gamma, \ldots, \Gamma^{(n)} / \Gamma\right)(z) \\
& \quad=\sum_{j=0}^{N} \frac{1}{\Gamma^{N-j}(z)} P_{j}\left(\zeta, \zeta^{\prime}, \ldots, \zeta^{(m)} ; 1, \Gamma^{\prime} / \Gamma, \ldots, \Gamma^{(n)} / \Gamma\right)(z) \not \equiv 0 .
\end{aligned}
$$

Now we estimate the term $P_{c, d}\left(\zeta, \zeta^{\prime}, \ldots, \zeta^{(m)} ; 1, \Gamma^{\prime} / \Gamma, \ldots, \Gamma^{(n)} / \Gamma\right)(z)$. It is known (e.g. [6, p. 151]) that

$$
\log \Gamma(z)=(z-1 / 2) \log z-z+\frac{1}{2} \log (2 \pi)+\int_{0}^{\infty} \frac{[u]-u+1 / 2}{u+z} d u .
$$

It follows that there is a $\delta>0$ such that

$$
\Gamma^{\prime}(z)=(1+o(1)) \Gamma(z) \log z
$$

uniformly for all $z \in \mathbb{C} \backslash\{z:|\arg z-\pi| \leq \delta\}$, where $o(1)$ stands for a 
quantity that goes to zero as $|z| \rightarrow \infty$. Similarly, for any positive integer $q$, there exists a $\delta>0$ such that

$$
\Gamma^{(q)}(z)=(1+o(1))(\log z)^{q} \Gamma(z)
$$

uniformly for all $z \in \mathbb{C} \backslash\{z:|\arg z-\pi| \leq \delta\}$. Thus, from (2) we find that

$$
\begin{aligned}
& P_{c, d}\left(\zeta, \zeta^{\prime}, \ldots, \zeta^{(m)} ; 1, \Gamma^{\prime} / \Gamma, \ldots, \Gamma^{(n)} / \Gamma\right)(z) \\
& =(\log z)^{d} \sum_{\lambda \in \Lambda_{c} \cap \Lambda_{d}^{*}} a_{\lambda}\left(\zeta, \zeta^{\prime}, \ldots, \zeta^{(m)}\right)(z)(1+o(1))^{\lambda_{1}} \cdots(1+o(1))^{\lambda_{n}}
\end{aligned}
$$

uniformly for all $z \in \mathbb{C} \backslash\{z:|\arg z-\pi| \leq \delta\}$.

Since $\sum_{\lambda \in \Lambda_{c} \cap \Lambda_{d}^{*}} a_{\lambda}\left(u_{0}, \ldots, u_{m}\right)$ is not identically zero, there are a $\delta_{0}>0$ and a bounded neighborhood $U$ of $w_{*}=\left(w_{0}, w_{1}, \ldots, w_{m}\right)$ such that

$$
\left|\sum_{\lambda \in \Lambda_{c} \cap \Lambda_{d}^{*}} a_{\lambda}\left(u_{0}, \ldots, u_{m}\right)\right| \geq \delta_{0} \quad \text { for } u=\left(u_{0}, u_{1}, \ldots, u_{m}\right) \in U \subset \mathbb{C}^{m+1} .
$$

By Voronin's lemma, there is a sequance $\left\{t_{q}\right\}_{q=1}^{\infty}$ of positive real numbers converging to $\infty$ such that

$$
\left(\zeta\left(3 / 4+i t_{q}\right), \zeta^{\prime}\left(3 / 4+i t_{q}\right), \ldots, \zeta^{(m)}\left(3 / 4+i t_{q}\right)\right) \in U
$$

for all $q=1,2, \ldots$ It follows from (5) that there are an $\varepsilon_{0}>0$ and $q_{0}$ such that, for $z_{q}=3 / 4+i t_{q}$,

$$
\left|P_{c, d}\left(\zeta, \zeta^{\prime}, \ldots, \zeta^{(m)} ; 1, \Gamma^{\prime} / \Gamma, \ldots, \Gamma^{(n)} / \Gamma\right)\left(z_{q}\right)\right| \geq \varepsilon_{0}\left|\log z_{q}\right|^{d}
$$

for all large $q \geq q_{0}$. If $d=0$, then (1) gives

$$
\begin{aligned}
\mid P_{c}\left(\zeta, \zeta^{\prime}, \ldots, \zeta^{(m)}\right. & \left.; 1, \Gamma^{\prime} / \Gamma, \ldots, \Gamma^{(n)} / \Gamma\right)\left(z_{q}\right) \mid \\
& =\left|P_{c, 0}\left(\zeta, \zeta^{\prime}, \ldots, \zeta^{(m)} ; 1, \Gamma^{\prime} / \Gamma, \ldots, \Gamma^{(n)} / \Gamma\right)\left(z_{q}\right)\right| \geq \varepsilon_{0}
\end{aligned}
$$

for all large $q$. If $d \geq 1$, then noting that $\zeta^{(p)}\left(z_{q}\right)$ is bounded for any $p$ and all large $q$, we see from (1) that

$$
\begin{aligned}
\left|P_{c}\left(\zeta, \zeta^{\prime}, \ldots, \zeta^{(m)} ; 1, \Gamma^{\prime} / \Gamma, \ldots, \Gamma^{(n)} / \Gamma\right)\left(z_{q}\right)\right| \\
\geq \varepsilon_{0}\left|\log z_{q}\right|^{d}-C\left|\log z_{q}\right|^{d-1} \rightarrow \infty \quad \text { as } q \rightarrow \infty,
\end{aligned}
$$

where $C$ is an absolute positive constant. Therefore, for any $d \geq 0$,

$$
\left|P_{c}\left(\zeta, \zeta^{\prime}, \ldots, \zeta^{(m)} ; 1, \Gamma^{\prime} / \Gamma, \ldots, \Gamma^{(n)} / \Gamma\right)\left(z_{q}\right)\right| \geq \varepsilon_{0},
$$

and, for any $d<p \leq N$,

$$
\left|P_{p}\left(\zeta, \zeta^{\prime}, \ldots, \zeta^{(m)} ; 1, \Gamma^{\prime} / \Gamma, \ldots, \Gamma^{(n)} / \Gamma\right)\left(z_{q}\right)\right| \leq C\left|\log z_{q}\right|^{M_{p}}
$$

for all large $q$. It is also known that (e.g. [6, p. 151]),

$$
|\Gamma(3 / 4+i y)| \sim e^{-\pi|y| / 2}|y|^{1 / 4} \sqrt{2 \pi}
$$

as $y \rightarrow \infty$. If $c<N$, we deduce from (4), (6) and (7) that 


$$
\begin{aligned}
& \left|\frac{P\left(\zeta, \zeta^{\prime}, \ldots, \zeta^{(m)} ; \Gamma, \Gamma^{\prime}, \ldots, \Gamma^{(n)}\right)\left(z_{q}\right)}{\Gamma^{N}\left(z_{q}\right)}\right| \\
& \quad=\left|\sum_{j=c}^{N} \frac{1}{\Gamma^{N-j}\left(z_{q}\right)} P_{j}\left(\zeta, \zeta^{\prime}, \ldots, \zeta^{(m)} ; 1, \Gamma^{\prime} / \Gamma, \ldots, \Gamma^{(n)} / \Gamma\right)\left(z_{q}\right)\right| \\
& \geq \varepsilon_{0}\left(\frac{e^{\pi t_{q} / 2}}{t_{q}^{1 / 4} \sqrt{2 \pi}}\right)^{N-c}-C\left|\log z_{q}\right|^{M_{N}}\left(\frac{e^{\pi t_{q} / 2}}{t_{q}^{1 / 4}}\right)^{N-c-1} \rightarrow \infty
\end{aligned}
$$

as $q \rightarrow \infty$, which completes the proof of the theorem in this case. If $c=N$, then

$$
\begin{aligned}
& P\left(\zeta, \zeta^{\prime}, \ldots, \zeta^{(m)} ; \Gamma, \Gamma^{\prime}, \ldots, \Gamma^{(n)}\right)\left(z_{q}\right) \\
& \quad=\Gamma^{N}\left(z_{q}\right) P_{N}\left(\zeta, \zeta^{\prime}, \ldots, \zeta^{(m)} ; 1, \Gamma^{\prime} / \Gamma, \ldots, \Gamma^{(n)} / \Gamma\right)\left(z_{q}\right) \neq 0
\end{aligned}
$$

for all large $q$, where we choose $z_{q}$ as in (6). Thus, the theorem is proved in this case. Therefore, we have completely proved the theorem.

COROLlary. If a non-trivial polynomial has the form $P\left(u_{0}, u_{1}, \ldots\right.$, $\left.u_{m} ; v_{0}, v_{1}\right)$, then

$$
P\left(\zeta, \zeta^{\prime}, \ldots, \zeta^{(m)} ; \Gamma, \Gamma^{\prime}\right)(z) \not \equiv 0
$$

Proof. For all possible $j$ 's and $p$ 's, the set $\Lambda_{j} \cap \Lambda_{p}^{*}$ only contains one element. So, the assumption in the theorem is satisfied. The corollary is proved.

\section{References}

[1] D. Hilbert, Die Hilbertschen Probleme, Geest \& Portig, Leipzig, 1979.

[2] O. Hölder, Über die Eigenschaft der $\Gamma$-Funktion, keiner algebraischen Differentialgleichung zu genügen, Math. Ann. 28 (1887), 1-13.

[3] L. Markus, Differential independence of $\Gamma$ and $\zeta$, J. Dynam. Differential Equations 19 (2007), 133-154.

[4] A. Ostrowski, Über Dirichlet Reihen und algebraische Differentialgleichungen, Math. Z. 8 (1920), 241-298.

[5] J. Steuding, Value Distribution of L-Functions, Lecture Notes in Math. 1877, Springer, New York, 2007.

[6] E. Titchmarsh, The Theory of Functions, Oxford Univ. Press, 1964.

[7] S. Voronin, On the distribution of nonzero values of Riemann's $\zeta$-function, Trudy Mat. Inst. Steklov. 128 (1972), 131-150.

Department of Mathematics

Florida International University

Miami, FL 33199, U.S.A.

E-mail: libaoqin@fiu.edu
Department of Mathematical Sciences

Northern Illinois University

DeKalb, IL 60115, U.S.A.

E-mail: ye@math.niu.edu 